\title{
Development of tricuspid regurgitation and right ventricular performance after implantation of centrifugal left ventricular assist devices
}

\author{
Johanna Mulzer ${ }^{1 \#}$, Hristo Krastev ${ }^{1 \#}$, Christoph Hoermandinger ${ }^{2}$, Alexander Meyer ${ }^{1}$, Thomas Haese ${ }^{2}$, \\ Julia Stein $^{2}$, Marcus Müller ${ }^{1}$, Felix Schoenrath ${ }^{1,3}$, Christoph Knosalla ${ }^{1,3}$, Christoph Starck ${ }^{1}$, \\ Volkmar Falk $^{1,3,4,5}$ Evgenij Potapov ${ }^{1}$ Jan Knierim ${ }^{1}$
}

${ }^{1}$ German Heart Center Berlin, Department of Cardiothoracic and Vascular Surgery, Berlin, Germany; ${ }^{2}$ DHZB Dienstleistungs GmbH, Berlin, Germany; ${ }^{3}$ DZHK (German Centre for Cardiovascular Research), Partner Site Berlin, Berlin, Germany; ${ }^{4}$ Department of Cardiothoracic Surgery, Charité Universitätsmedizin Berlin, corporate member of Freie Universität Berlin, Humboldt-Universität zu Berlin, and Berlin Institute of Health, Berlin, Germany; ${ }^{5}$ Eidgenössiche Technische Hochschule Zürich, Department of Health Sciences and Technology, Translational Cardiovascular Technology, Zurich, Switzerland

\#These authors contributed equally to this work.

Correspondence to: Dr. med. Jan Knierim. Deutsches Herzzentrum Berlin, Klinik für Herz-, Thorax- und Gefäßchirurgie, Augustenburger Platz 1, 13353 Berlin, Germany. Email: knierim@dhzb.de.

Background: Tricuspid regurgitation (TR) after left ventricular assist device (LVAD) implantation is associated with a poor prognosis. This study evaluates the development of TR and right ventricular (RV) performance after LVAD implantation.

Methods: Retrospective analysis of patients who underwent LVAD implantation between March 2018 and June 2019. Patients who underwent concomitant tricuspid valve surgery and patients with congenital heart disease were excluded.

Results: A total of 155 patients underwent LVAD implantation. Fourteen patients were excluded. Of the remaining patients, thirty-one died during the first six months, six were lost to follow-up and two underwent transplantation. 102 patients presented at 6.3 months (5.8 to 7.0). Patients were supported with HeartWare HVAD (74\%) or HeartMate 3 (26\%). 50.4\% were rated as INTERMACS profile 1 or 2. At six months, systolic pulmonary artery pressure dropped from 36 to $21 \mathrm{mmHg}(\mathrm{P}<0.001)$. Tricuspid annular plane systolic excursion decreased from 17.3 to $14.3 \mathrm{~mm}(\mathrm{P}<0.001)$, RV fractional area change did not change $(\mathrm{P}=0.839)$. Twenty-two patients (22\%) presented with moderate-to-severe or severe (ms-s) TR pre-operatively. Of these, eighteen $(81 \%)$ showed improvement to $\leq$ moderate TR. At follow-up twelve patients presented with ms-s TR. Of these, only four patients (33\%) had been diagnosed with ms-s TR pre-operatively. There were no differences in pre-operative echocardiographic or clinical parameters between the twelve patients with ms-s late TR and the other ninety patients in the cohort.

Conclusions: TR can show an impressive improvement with LVAD support. Longitudinal RV function decreases; this appears to be compensated by transverse shortening. Late TR can develop independently from pre-operative parameters including TR.

Keywords: Left ventricular assist device (LVAD); tricuspid regurgitation; right ventricular function

Submitted Oct 11, 2020. Accepted for publication Feb 08, 2021.

doi: 10.21037/acs-2020-cfmcs-fs-0215

View this article at: http://dx.doi.org/10.21037/acs-2020-cfmcs-fs-0215 


\section{Introduction}

The implantation of centrifugal left ventricular assist devices (LVAD) for the treatment of advanced heart failure has increased significantly in recent years (1). The latest generation of centrifugal LVADs yield excellent results with regard to a lower complication profile, functional status and quality of life $(2,3)$. Right heart failure (RHF) remains a challenging and sometimes unpredictable problem, which limits the prognosis of patients with LVADs. Pre-operative tricuspid regurgitation (TR) is associated with poor survival and may be a risk factor for early RHF $(4,5)$. It is also a predictor for the development of RHF following LVAD implantation (6). Residual TR after LVAD implantation is associated with higher mortality (7).

Therefore, some have suggested to concomitantly perform tricuspid valve repair (TVR) in patients with more than moderate TR undergoing LVAD implantation. In contemporary guidelines this approach is described as "generally accepted" or recommended restrictively as it "may be considered in carefully selected patients (IIb)" $(8,9)$. The clinical benefits supporting such combined procedures are still unclear. Some single-center studies showed a reduction in post-operative RHF or readmission rates in patients undergoing concomitant TVR while others could not confirm these findings (10-13). In retrospective multicenter registries, TVR at the time of LVAD implantation was not associated with improved survival $(4,14)$.

It has been shown that LVAD implantation decreases pulmonary arterial pressure, central venous pressure and TR $(15,16)$. However, about $10 \%$ of patients present with moderate-to-severe TR one year after LVAD implantation, regardless of whether they underwent TVR or not (14).

The aim of this study was to evaluate the clinical course of TR and echocardiographic parameters of right ventricular size and function in patients undergoing centrifugal LVAD implantation without TVR.

\section{Methods}

\section{Study population}

For this study, the records of all patients who had undergone LVAD implantation between March 2018 and June 2019 were reviewed. Since June 2018, all patients who presented in the outpatient unit of our center six months after LVAD implantation underwent a standard evaluation including an interview, physical examination, laboratory tests, ECG, six-minute walk test and a standardized echocardiogram. Patients who underwent concomitant tricuspid valve repair (TVR), patients who underwent permanent biventricular assist device implantation and patients with congenital heart disease were excluded.

All data were collected in a RedCap database and analyzed retrospectively. The study was reviewed and approved by the ethics committee at Charité University (EA2/229/19).

\section{Surgical technique}

In most cases, LVAD implantation was performed via median sternotomy. In patients with previous cardiac surgery and a status post sternotomy, LVAD implantation was performed via left lateral thoracotomy. If concomitant intracardiac procedures (e.g., valve surgery, left ventricular thrombectomy or patent foramen ovale/atrial septal defect (PFO/ASD) closure) were necessary, the implantation was performed employing cardiopulmonary bypass. Regarding tricuspid surgery, our institution has followed a very conservative policy for more than fifteen years. In our opinion, only very few cases justify concomitant TV surgery. These are:

* Structural damage of the TV causing severe TR;

* Severe tethering of TV leaflets;

* Severe functional TR regurgitation and a concomitant need to open the right atrium (e.g., for PFO/ASD closure or removal of infected leads from cardiac implantable electrical devices).

In the case of TV repair, the double orifice technique was preferred (17). In the case of severe tethering, TV replacement was considered the technique of choice.

If no concomitant intracardiac procedure was required, the LVAD implantation was primarily carried out using off-pump techniques; however, in cases of hemodynamic instability, circulatory support was provided by extracorporeal life support (ECLS). In patients already on temporary circulatory support (ECLS or Impella ${ }^{\circledR}$ ), the support was continued during surgery, except when intracardiac procedures were necessary, in which case the circulatory support was switched to cardiopulmonary bypass.

In the case of severe peri-operative RHF, a temporary right ventricular assist device (RVAD) was implanted and the right ventricle was supported for 10-14 days. In the case of RHF plus severe TR, we believe that the TR is 
usually functional and caused by the right ventricular failure. Therefore, TV repair was not used to wean from cardiopulmonary bypass or RVAD. If RV failure and TR did not improve during temporary mechanical support, the RVAD was switched to a durable device in a second step.

\section{Device and patient management}

The device speed was clinically adjusted to optimize flow and organ perfusion prior to discharge and during every outpatient visit. During the hospital stay and in later followup, echocardiography was performed serially to evaluate the position of the interventricular septum as well as the left ventricular (LV) and right ventricular (RV) size and function. Maintenance of intermittent opening of the aortic valve was per intention by adjusting device speed. Anticoagulation with warfarin plus antiplatelet therapy was administered as recommended by current guidelines $(8,9)$. All patients received heart failure medication as recommended by the European Society of Cardiology (ESC) heart failure guidelines (18). Management of blood pressure included a target mean arterial pressure lower than $90 \mathrm{mmHg}$. Loop diuretics were given in case of fluid overload.

\section{Echocardiography}

Pre-operative transthoracic echocardiograms obtained within five days prior to surgery were reviewed. If preoperative images were not available, the results of external echocardiograms were taken $(n=5)$. At the six-month followup, all patients underwent a standardized echocardiography at our institution. The echocardiography was performed by experienced sonographers as per the current guidelines and recommendations (19-21).

\section{Statistical analysis}

For categorical data, frequencies and percentages are reported. Patient groups were compared using the Fisher's exact test or chi-squared test.-

Continuous data are summarized as mean and standard deviation (SD) or, in cases of skewed data, as median and interquartile range (IQR). Patient groups were compared using Student's $t$-test or the Mann-Whitney U test according to the variables' distribution. Comparisons prior to surgery and after six months were performed using a paired $t$-test.

We assumed $\mathrm{P}<0.05$ as the threshold for statistical significance. Analyses were exploratory in nature. For all statistical analysis, we used R software, Version 3.5.2.

\section{Results}

\section{Baseline characteristics}

A total of 155 records were reviewed. Patients who underwent concomitant tricuspid valve surgery $(n=12)$ and patients with congenital heart disease $(n=2)$ were excluded. LVAD implantation was performed via median sternotomy $(n=126,89 \%)$ or lateral thoracotomy $(n=15$, $11 \%)$. Pre-operative mechanical circulatory support was provided by ECLS $(n=26,18 \%)$ or Impella ${ }^{\circledR}(n=18$, $13 \%)$. Intra-operative circulatory support by ECLS or Impella ${ }^{\circledR}$ was provided resp. continued $52 \%$ of the cases. Cardiopulmonary bypass was employed in twenty-nine patients (21\%).

Thirty-one (22\%) patients died during the first six (4\%) months, six were lost to follow-up and two underwent transplantation.

102 patients presented at median 6.25 months (range 5.88 to 7.04 ) for the standardized follow-up visit. They were supported with either HeartWare HVAD $(n=72)$ or HeartMate $3(n=30)$ devices. For pre-operative characteristics see Table 1 . Post-operatively all patients received optimal heart failure medication including betablockers $(87 \%)$, aldosterone antagonists $(84 \%)$, and inhibition of the renin-angiotensin system (96\%). Most of the patients were in a good physical condition and were classified as NYHA I or II (82\%). For device settings and further details see Table 2.

\section{Comparison between patients who presented for follow-up and patients who died during the first six months}

The thirty-one patients who died during the first six months had a significantly longer duration of heart failure $(\mathrm{P}=0.047)$ and a higher INTERMACS profile $(\mathrm{P}=0.021)$. Also Rethoracotomy [ $\mathrm{n}=16$ (42\%) vs. $\mathrm{n}=30$ (15.7\%), $\mathrm{P}=0.001]$ and temporary $\mathrm{RV}$ assist device implantation $[\mathrm{n}=15$ $(42 \%) v s . \mathrm{n}=2(2 \%), \mathrm{P}<0.001]$ was required more often in these patients. The grade of pre-operative TR did not differ significantly between the patients who died and those who presented at six months $(\mathrm{P}=0.168)$. For details see Table 1 . 
Table 1 Preoperative and operative characteristics $(\mathrm{n}=141)$



CRT, cardiac resynchronization therapy; CRT-D, cardiac resynchronization therapy defibrillator; ICD, implantable cardioverter-defibrillator; INTERMACS, Interagency Registry for Mechanical Assisted Devices; IQR, interquartile range; SD, standard deviation. 


\begin{tabular}{|c|c|}
\hline Characteristics & Value \\
\hline \multicolumn{2}{|l|}{ Device settings HeartWare $(n=72)$} \\
\hline Rounds per minute, mean (SD) & $2,650.00(150.1)$ \\
\hline Flow (L/min), mean (SD) & $4.82(0.6)$ \\
\hline Power $(\mathrm{W})$, mean $(\mathrm{SD})$ & $4.22(4.8)$ \\
\hline \multicolumn{2}{|l|}{ Device settings HeartMate $3(n=30)$} \\
\hline Rounds per minute, mean (SD) & $5,506.67(282.5)$ \\
\hline Flow (L/min), mean (SD) & $4.63(0.4)$ \\
\hline Power (W), mean (SD) & $4.24(0.5)$ \\
\hline \multicolumn{2}{|l|}{ NYHA class, n (\%) } \\
\hline NYHA I & $47(47.0)$ \\
\hline NYHA II & $35(35.0)$ \\
\hline NYHA III & $18(18.0)$ \\
\hline NYHA IV & $0(0.0)$ \\
\hline Mean arterial pressure $(\mathrm{mmHg})$, mean (SD) & $82.34(11.1)$ \\
\hline Heart rate, mean (SD) & $75.78(15.8)$ \\
\hline Atrial fibrillation, n (\%) & $14(14.3)$ \\
\hline \multicolumn{2}{|l|}{ Heart failure medication, $\mathrm{n}(\%)$} \\
\hline Beta-blockers & $89(87.3)$ \\
\hline Angiotensin-converting enzyme inhibitor & $16(15.8)$ \\
\hline Angiotensin II receptor blocker & $16(15.7)$ \\
\hline Angiotensin-receptor neprilysin inhibitor & $66(64.7)$ \\
\hline Aldosterone antagonists & $86(84.3)$ \\
\hline Loop diuretics & $78(77.2)$ \\
\hline
\end{tabular}

NYHA, New York Heart Functional Classification; SD, standard deviation.

\section{Echocardiographic changes of the right heart under cLVAD support}

Six months after implantation, left ventricular unloading had led to a considerable decrease in systolic pulmonary artery pressure from $36[13]$ to $21[6] \mathrm{mmHg}(\mathrm{P}<0.001)$. There was a slight but significant reduction in the basal right ventricular diameter from 44 [7] to 40 [8] $\mathrm{mm}(\mathrm{P}=0.002)$, but no significant change in the proximal right ventricular outflow tract diameter (proxRVOT) and right ventricular end-diastolic area (RV-EDA). The tricuspid annular plane systolic excursion (TAPSE), representing longitudinal right ventricular function, decreased significantly, while the right ventricular fractional area change did not change, and the visual RV ejection fraction improved slightly (Table 3).

\section{Development of tricuspid regurgitation}

In total an impressive decrease in TR could be demonstrated in patients who underwent echocardiography six months after LVAD implantation. $55 \%$ of the patients showed more than mild TR pre-operatively and only $27 \%$ at follow up (Figure 1).

Twenty-two patients presented with moderate-to-severe or severe (ms-s) TR pre-operatively. Of these, eighteen $(81 \%)$ showed a significant improvement to $\leq$ moderate TR (Figure 2).

In comparison, twelve patients presented with ms-s TR at the six-month visit. However, of these, only four (33\%) had been diagnosed with ms-s TR before the LVAD implantation. Therefore, eight patients showed new onset of ms-s TR after LVAD implantation (Figure 2).

\section{Predictors of late TR}

No significant differences were found in pre-operative or operative parameters between the twelve patients with ms-s TR at the six-month visit compared to all patients with $\leq$ moderate TR ( $\mathrm{n}=90)$. Also, pre-operative echocardiography showed no significant difference between the two groups; see Table 4.

\section{Discussion}

Our study demonstrates that in the majority of patients, pre-operative TR improves with left ventricular unloading following the implantation of an LVAD (Figure 1). In some patient's late TR may develop during the follow-up whilst on LVAD.

With an incidence ranging between $10 \%$ and $50 \%$, preoperative ms-s TR is very common in patients undergoing LVAD implantation $(4,7,10,16)$. Relevant pre-operative TR is associated with a poorer survival and may contribute to early and/or late right heart failure after LVAD implantation (4-7). As a result, concomitant TVR has been discussed as a logical consequence; the clinical benefits of this procedure are still unclear $(4,10,11,14)$. In the current STS/ISHLT expert opinion, TVR is described as "generally accepted" but is not specifically recommended, while the EACTS expert consensus states that TVR "may be considered in carefully selected patients (IIb)” (8,9). 


\begin{tabular}{|c|c|c|c|}
\hline Left ventricular end-diastolic diameter (mm), mean (SD) & $67.0(10.9)$ & $55.7(12.9)$ & $<0.001$ \\
\hline proxRVOT (mm), mean (SD) & $35.9(7.02)$ & $34.6(6.0)$ & 0.054 \\
\hline Basal right ventricular diameter (mm), mean (SD) & $43.6(7.3)$ & $40.0(8.0)$ & 0.002 \\
\hline RV fractional area change (\%), mean (SD) & $29.2(9.7)$ & $29.8(13.7)$ & 0.839 \\
\hline TAPSE (mm), mean (SD) & $17.3(3.9)$ & $14.3(3.8)$ & $<0.001$ \\
\hline Systolic pulmonary artery pressure $(\mathrm{mmHg})$, mean (SD) & $35.7(12.6)$ & $21.4(5.9)$ & $<0.001$ \\
\hline Tricuspid valve annulus (mm), mean (SD) & $37.6(6.4)$ & $34.3(5.2)$ & $<0.001$ \\
\hline
\end{tabular}

proxRVOT, proximal right ventricular outflow tract diameter; RV, right ventricular; RV-EDA, right ventricular end-diastolic area; TAPSE, tricuspid annular plane systolic excursion.

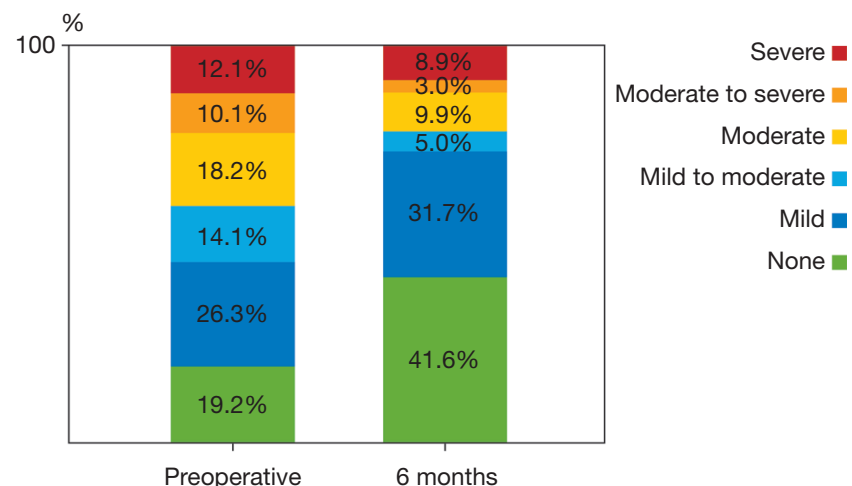

Figure 1 Clinical course of tricuspid regurgitation in all patients that presented at 6 months for the standardized follow-up visit. Left: preoperative degree of TR ( $\mathrm{n}=99$, missing $\mathrm{n}=3)$; right: six months after LVAD implantation ( $\mathrm{n}=102)$.

There are many publications that deal with acute right $\mathrm{RV}$ failure accompanied by TR in the early postoperative phase $(5,22,23)$. In our cohort, early RV failure temporarily requiring an $\mathrm{RV}$ assist device was also frequent in patients who did not survive the first six months (Table 1). However, the incidence and grade of pre-operative tricuspid regurgitation did not differ significantly between survivors and non-survivors. The focus of our work was to analyze the development of RV failure and TR in post-operative period. This topic is of particular importance since right heart failure is one of the major causes of death in the long term, in patients with left ventricular assist devices $(24,25)$.

LVAD implantation decompresses the pulmonary

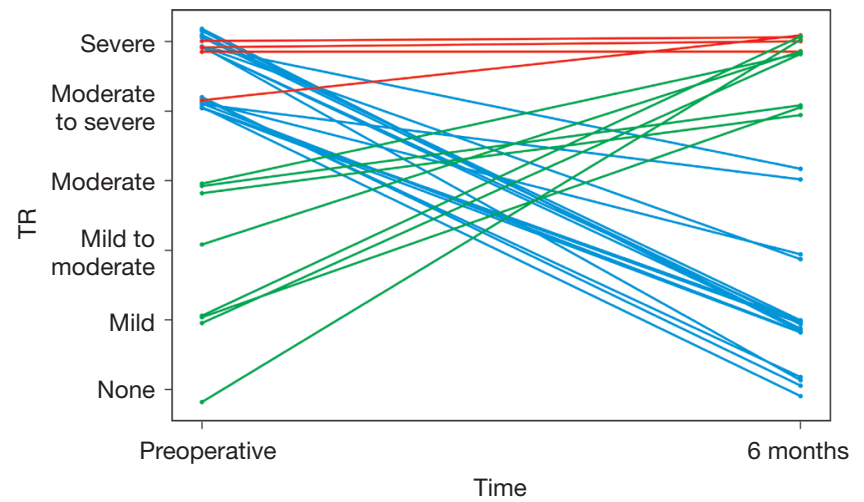

Figure 2 Clinical course of tricuspid regurgitation (TR) in all patients with preoperative moderate-to-severe or severe (ms-s) TR $(\mathrm{n}=22)$ and/or ms-s TR at six months $(\mathrm{n}=12)$. Left: preoperative degree of TR; right: TR in the same patients six months after LVAD implantation. Blue lines: patients with TR improvement. Green lines: patients with new ms-s TR. Red lines: patients with ms-s TR preoperatively and at six months.

circulation and subsequently decreases pulmonary artery pressure (15). This may lead to reverse RV remodeling $(15,16)$. Despite a decrease in systolic pulmonary artery pressure in our patients, no reverse RV remodeling occurred except for a decrease in the basal RV diameter and the tricuspid annulus diameter (Table 3). This may be one contributing factor to an improvement in TR under LVAD support. An increase in right ventricular function has been described by some authors. Morgan et al. saw an improvement in TAPSE and right ventricular ejection 
fraction and Atluri et al. showed a significant increase in RV stroke work index $(15,16)$. We found a decrease in the right ventricular longitudinal function as reflected by TAPSE. This may not represent the global function and may be compensated by an increase in transverse shortening (26), since we saw no changes in right ventricular fractional area change and a slight improvement in the visually estimated right ventricular ejection fraction (Table 3).

Pre-operative tricuspid regurgitation improved in most of our patients (Figure 1). Eighteen of twenty-two patients with severe or moderate-to-severe pre-operative TR (81\%) had $\leq$ moderate TR at the 6-month follow-up visit. Of these, sixteen (72\%) showed an improvement to < moderate
TR (Figure 2).

Only four $(33 \%)$ of the twelve patients with late TR had had moderate-to-severe or severe TR before LVAD implantation. The other eight patients developed new TR after the implantation (Figure 2). This finding, together with the improvement in pre-operative TR, may partially explain why concomitant TVP does not improve survival in large registries $(4,14)$.

Pre-operative atrial fibrillation may be associated with early progression of TR after LVAD implantation (27). In our study, the incidence of atrial fibrillation did not differ between patients with or without late TR after LVAD implantation (Table 4). Leads of cardiac implantable

Table 4 Comparison between patients with moderate-to-severe or severe (ms-s) TR vs. patients with $\leq$ moderate TR

\begin{tabular}{|c|c|c|c|}
\hline & $\leq$ moderate $T R(n=90)$ & ms-s TR ( $n=12)$ & $P$ value \\
\hline \multicolumn{4}{|l|}{ Preoperative parameters } \\
\hline Age (years), mean (SD) & $55.7(11.0)$ & $58.2(14.0)$ & 0.485 \\
\hline Male, n (\%) & 78 (86.7) & $9(75.0)$ & 0.284 \\
\hline Body mass index $\left(\mathrm{kg} / \mathrm{m}^{2}\right)$, mean $(\mathrm{SD})$ & $27.2(5.8)$ & $30.1(6.1)$ & 0.123 \\
\hline Diabetes & $32(31.2)$ & $3(25.0)$ & 0.895 \\
\hline Hypertension & $59(65.6)$ & $6(50.0)$ & 0.326 \\
\hline Glomerular filtration rate $(\mathrm{mL} / \mathrm{min})$, median (IQR) & 58.7 (39.2 to 85.9$)$ & 54.4 (43.1 to 60.9$)$ & 0.518 \\
\hline \multicolumn{4}{|l|}{ Etiology of heart failure, $n(\%)$} \\
\hline Dilated cardiomyopathy & $42(46.7)$ & $5(41.7)$ & 0.239 \\
\hline Ischemic cardiomyopathy & $44(48.9)$ & $5(41.7)$ & \\
\hline Other cardiomyopathy & $4(4.4)$ & $2(16.7)$ & \\
\hline Duration of heart failure (months), median (IQR) & 72.0 (3.0 to 147.8$)$ & $20.5(2.5$ to 40.5$)$ & 0.164 \\
\hline Known atrial fibrillation, $\mathrm{n}(\%)$ & $38(42.2)$ & $5(41.7)$ & 1 \\
\hline Preoperative atrial fibrillation, $\mathrm{n}(\%)$ & $29(32.2)$ & $4(33.3)$ & 1 \\
\hline \multicolumn{4}{|l|}{ Device, n (\%) } \\
\hline ICD & $32(35.6)$ & $3(25.0)$ & 0.594 \\
\hline CRT & $3(3.3)$ & $1(8.3)$ & \\
\hline CRT-D & $22(24.4)$ & $3(25.0)$ & \\
\hline \multicolumn{4}{|l|}{ INTERMACS profile, $\mathrm{n}(\%)$} \\
\hline INTERMACS 1 & $19(21.1)$ & $5(41.7)$ & 0.531 \\
\hline INTERMACS 2 & 20 (22.2) & $3(25.0)$ & \\
\hline INTERMACS 3 & $21(23.3)$ & $2(16.7)$ & \\
\hline INTERMACS 4 & $28(31.1)$ & $2(16.7)$ & \\
\hline INTERMACS 5 & $2(2.2)$ & $0(0.0)$ & \\
\hline
\end{tabular}

Table 4 (continued) 
Table 4 (continued)

\begin{tabular}{|c|c|c|c|}
\hline & $\leq$ moderate TR $(n=90)$ & ms-s TR $(n=12)$ & $P$ value \\
\hline Previous cardiac surgery, $\mathrm{n}(\%)$ & $13(86.7)$ & $2(13.3)$ & 0.838 \\
\hline \multicolumn{4}{|l|}{ Device, n (\%) } \\
\hline HeartWare HVAD & $63(70.0)$ & $9(75.0)$ & 0.721 \\
\hline Temporary RV assist device, $\mathrm{n}(\%)$ & $1(1.1)$ & $1(8.3)$ & 0.573 \\
\hline Rethoracotomy, n (\%) & $15(16.7)$ & $1(8.3)$ & 0.747 \\
\hline \multicolumn{4}{|l|}{ LVAD settings } \\
\hline \multicolumn{4}{|l|}{ HeartWare HVAD ( $n=72)$} \\
\hline Power (W), mean (SD) & $4.2(0.7)$ & $4.4(0.7)$ & 0.371 \\
\hline \multicolumn{4}{|l|}{ HeartMate $3(n=30)$} \\
\hline Rounds per minute, mean (SD) & $5,522.2(278.3)$ & $5,366.7(208.2)$ & 0.373 \\
\hline Flow (L/min), mean (SD) & $4.7(0.4)$ & $4.2(0.3)$ & 0.051 \\
\hline Power (W), mean (SD) & $4.27(0.3)$ & $4.1(0.5)$ & 0.547 \\
\hline \multicolumn{4}{|l|}{ Preoperative echocardiography } \\
\hline Left ventricular end-diastolic diameter (mm) & $67.0(11.0)$ & $67.3(11.4)$ & 0.916 \\
\hline Left ventricular end-diastolic volume (mL), mean (SD) & $257.6(101.7)$ & $293.4(134.4)$ & 0.339 \\
\hline RV ejection fraction (\%), mean (SD) & $44.6(9.3)$ & $42.3(9.3)$ & 0.428 \\
\hline TAPSE (mm), mean (SD) & $17.3(3.8)$ & $15.6(4.6)$ & 0.333 \\
\hline Systolic pulmonary artery pressure $(\mathrm{mmHg})$, mean (SD) & 34.7 (12.2) & $32.4(9.5)$ & 0.554 \\
\hline \multicolumn{4}{|l|}{ Mitral regurgitation, n (\%) } \\
\hline None & $5(5.9)$ & $2(16.7)$ & 0.398 \\
\hline Mild & $23(27.1)$ & $2(16.7)$ & \\
\hline Mild to moderate & $13(15.3)$ & $0(0.0)$ & \\
\hline Moderate & $14(16.5)$ & $2(16.7)$ & \\
\hline Moderate to severe & $9(10.6)$ & $1(8.3)$ & \\
\hline Severe & $21(24.7)$ & $5(41.7)$ & \\
\hline
\end{tabular}




\begin{tabular}{|c|c|c|c|}
\hline & $\leq$ moderate TR $(n=90)$ & ms-s TR $(n=12)$ & $P$ value \\
\hline \multicolumn{4}{|c|}{ Tricuspid regurgitation, $\mathrm{n}(\%)$} \\
\hline None & $18(20.7)$ & $1(8.3)$ & 0.634 \\
\hline Mild & $23(26.4)$ & $3(25.0)$ & \\
\hline Mild to moderate & $13(14.9)$ & $1(8.3)$ & \\
\hline Moderate & $15(17.2)$ & $3(25.0)$ & \\
\hline Moderate to severe & $9(10.3)$ & $1(8.3)$ & \\
\hline Severe & $9(10.3)$ & $3(25.0)$ & \\
\hline
\end{tabular}

electronic devices can cause TR as well. However, there was no significant difference in the number of pacemaker, ICD or CRT devices between patients with or without late TR in our cohort.

Echocardiography is generally used to assess right ventricular function and to predict early right ventricular failure (22,28-30). However, there is little knowledge about the predictive value of pre-operative echocardiography regarding late right heart failure and late tricuspid regurgitation. The only preoperative parameter that has been identified by other groups as a predictor for late TR is the tricuspid annulus diameter (31). We were unable to reproduce this result in our admittedly smaller study (Table 3). Also, all other pre-operative echocardiographic parameters of left and right ventricular size and function did not differ significantly between the patients with or without late significant TR (Table 4).

\section{Limitations}

The most effective way to identify predictors of significant TR at six months would be a multivariable analysis or propensity matching. Unfortunately, the sample size and event rate (development of late TR) of our study was too small for this, and further analysis of larger registries is required. We were, however, able to show that pre-operative TR or a limited number of additional echocardiographic measures are not able to predict the later course of RV function and TR development.

\section{Conclusions}

Our study demonstrates that LVAD implantation causes left ventricular unloading and a decrease in pulmonary artery pressure. Longitudinal right ventricular function represented by TAPSE decreases, but this seems to be compensated by transverse shortening of the RV. TR usually shows an impressive improvement. However, in some cases new TR develops after the implantation. This may happen in patients with normal pre-operative tricuspid valve function.

\section{Acknowledgments}

Funding: None.

\section{Footnote}

Conflicts of Interest: Volkmar Falk is a member of the advisory boards of Abbott, Medtronic and Berlin Heart, and a principal investigator for $\mathrm{ZH}$ Heart. Evgenij Potapov reports grants from Abbott and Medtronic and professional fees as a proctor for Abbott and Medtronic. Felix Schoenrath receives remuneration, consultancy fees and/ or travel support from Medtronic, Abbott, Cardiorentis, and a research grant from Novartis. Christoph Starck reports grants from Cook Medical, Abbott, Medtronic and Biotronic and personal fees from Biotronic, Angiodynamics and Liva Nova. Alexander Meyer reports grants from Berlin Institute of Health, Edwards Lifesciences, Abbott and Maquet and grants and other support from Medtronic. The other authors have no conflicts of interest to disclose.

Open Access Statement: This is an Open Access article 
distributed in accordance with the Creative Commons Attribution-NonCommercial-NoDerivs 4.0 International License (CC BY-NC-ND 4.0), which permits the noncommercial replication and distribution of the article with the strict proviso that no changes or edits are made and the original work is properly cited (including links to both the formal publication through the relevant DOI and the license). See: https://creativecommons.org/licenses/by-nc-nd/4.0/.

\section{References}

1. Fernandez FG, Shahian DM, Kormos R, et al. The Society of Thoracic Surgeons National Database 2019 Annual Report. Ann Thorac Surg 2019;108:1625-32.

2. Mehra MR, Uriel N, Naka Y, et al. A Fully Magnetically Levitated Left Ventricular Assist Device - Final Report. N Engl J Med 2019;380:1618-27.

3. Rogers JG, Pagani FD, Tatooles AJ, et al. Intrapericardial Left Ventricular Assist Device for Advanced Heart Failure. N Engl J Med 2017;376:451-60.

4. Song HK, Gelow JM, Mudd J, et al. Limited Utility of Tricuspid Valve Repair at the Time of Left Ventricular Assist Device Implantation. Ann Thorac Surg 2016;101:2168-74.

5. Lampert BC, Teuteberg JJ. Right ventricular failure after left ventricular assist devices. J Heart Lung Transplant 2015;34:1123-30.

6. Gonzalez-Fernandez O, Bouzas-Cruz N, Ferrera C, et al. Effect of Preoperative Tricuspid and/or Mitral Regurgitation on Development of Late Right-Sided Heart Failure After Insertion of the HeartWare Left Ventricular Assist Device. Am J Cardiol 2020;125:236-43.

7. Nakanishi K, Homma S, Han J, et al. Prevalence, Predictors, and Prognostic Value of Residual Tricuspid Regurgitation in Patients With Left Ventricular Assist Device. J Am Heart Assoc 2018;7:e008813.

8. Kirklin JK, Pagani FD, Goldstein DJ, et al. American Association for Thoracic Surgery/International Society for Heart and Lung Transplantation guidelines on selected topics in mechanical circulatory support. J Heart Lung Transplant 2020;39:187-219.

9. Potapov EV, Antonides C, Crespo-Leiro MG, et al. 2019 EACTS Expert Consensus on long-term mechanical circulatory support. Eur J Cardiothorac Surg 2019;56:230-70.

10. Piacentino V, Williams ML, Depp T, et al. Impact of Tricuspid Valve Regurgitation in Patients Treated With Implantable Left Ventricular Assist Devices. Ann Thorac
Surg 2011;91:1342-6; discussion 1346-7.

11. Critsinelis A, Kurihara C, Kawabori M, et al. Outcomes in patients who underwent a concomitant tricuspid valve procedure during left ventricular assist device implantation. J Card Surg 2019;34:1458-64.

12. Oezpeker C, Zittermann A, Paluszkiewicz L, et al. Tricuspid valve repair in patients with left-ventricular assist device implants and tricuspid valve regurgitation: propensity score-adjusted analysis of clinical outcome. Interact Cardiovasc Thorac Surg 2015;21:741-7.

13. Saeed D, Kidambi T, Shalli S, et al. Tricuspid valve repair with left ventricular assist device implantation: Is it warranted? J Heart Lung Transplant 2011;30:530-5.

14. Veen KM, Caliskan K, de By TMMH, et al. Outcomes after tricuspid valve surgery concomitant with left ventricular assist device implantation in the EUROMACS registry: a propensity score matched analysis. Eur J Cardiothorac Surg 2019;56:1081-9.

15. Atluri P, Fairman AS, MacArthur JW, et al. Continuous Flow Left Ventricular Assist Device Implant Significantly Improves Pulmonary Hypertension, Right Ventricular Contractility, and Tricuspid Valve Competence: LVAD IMPROVES PULMONARY HYPERTENSION AND RV FUNCTION. J Card Surg 2013;28:770-5.

16. Morgan JA, Paone G, Nemeh HW, et al. Impact of continuous-flow left ventricular assist device support on right ventricular function. J Heart Lung Transplant 2013;32:398-403.

17. Hetzer R, Javier M, Delmo Walter EM. The DoubleOrifice Valve Technique to Treat Tricuspid Valve Incompetence. J Heart Valve Dis 2016;25:66-71.

18. Ponikowski P, Voors AA, Anker SD, et al. 2016 ESC Guidelines for the diagnosis and treatment of acute and chronic heart failure: The Task Force for the diagnosis and treatment of acute and chronic heart failure of the European Society of Cardiology (ESC)Developed with the special contribution of the Heart Failure Association (HFA) of the ESC. Eur Heart J 2016;37:2129-2200.

19. Zoghbi WA, Adams D, Bonow RO, et al. Recommendations for Noninvasive Evaluation of Native Valvular Regurgitation. J Am Soc Echocardiogr 2017;30:303-71.

20. Lang RM, Badano LP, Mor-Avi V, et al. Recommendations for Cardiac Chamber Quantification by Echocardiography in Adults: An Update from the American Society of Echocardiography and the European Association of Cardiovascular Imaging. Eur Heart J Cardiovasc Imaging 2015;16:233-70. 
21. Stainback RF, Estep JD, Agler DA, et al. Echocardiography in the Management of Patients with Left Ventricular Assist Devices: Recommendations from the American Society of Echocardiography. J Am Soc Echocardiogr 2015;28:853-909.

22. Dandel M, Krabatsch T, Falk V. Left ventricular vs. biventricular mechanical support: Decision making and strategies for avoidance of right heart failure after left ventricular assist device implantation. Int J Cardiol 2015;198:241-50.

23. Loghmanpour NA, Kormos RL, Kanwar MK, et al. A Bayesian Model to Predict Right Ventricular Failure Following Left Ventricular Assist Device Therapy. JACC Heart Fail 2016;4:711-21.

24. Dunlay SM, Deo SV, Park SJ. Impact of Tricuspid Valve Surgery at the Time of Left Ventricular Assist Device Insertion on Postoperative Outcomes. ASAIO J 2015;61:15-20.

25. Stulak JM, Mehta V, Schirger JA, et al. Temporal Differences in Causes of Mortality After Left Ventricular Assist Device Implantation. Ann Thorac Surg 2015;99:1969-72; discussion 1972-4.

26. Raina A, Vaidya A, Gertz ZM, et al. Marked changes in right ventricular contractile pattern after cardiothoracic surgery: Implications for post-surgical assessment of right ventricular function. J Heart Lung Transplant

Cite this article as: Mulzer J, Krastev H, Hoermandinger C, Meyer A, Haese T, Stein J, Müller M, Schoenrath F, Knosalla C, Starck C, Falk V, Potapov E, Knierim J. Development of tricuspid regurgitation and right ventricular performance after implantation of centrifugal left ventricular assist devices. Ann Cardiothorac Surg 2021;10(3):364-374. doi: 10.21037/acs-2020cfmcs-fs-0215
2013;32:777-83.

27. Anwer LA, Tchantchaleishvili V, Poddi S, et al. Atrial Fibrillation Should Guide Prophylactic Tricuspid Procedures During Left Ventricular Assist Device Implantation. ASAIO J 2018;64:586-93.

28. Aissaoui N, Salem JE, Paluszkiewicz L, et al. Assessment of right ventricular dysfunction predictors before the implantation of a left ventricular assist device in end-stage heart failure patients using echocardiographic measures (ARVADE): Combination of left and right ventricular echocardiographic variables. Arch Cardiovasc Dis 2015;108:300-9.

29. Kukucka M, Stepanenko A, Potapov E, et al. Right-toleft ventricular end-diastolic diameter ratio and prediction of right ventricular failure with continuous-flow left ventricular assist devices. J Heart Lung Transplant 2011;30:64-9.

30. Topilsky Y, Oh JK, Shah DK, et al. Echocardiographic Predictors of Adverse Outcomes After Continuous Left Ventricular Assist Device Implantation. JACC Cardiovasc Imaging 2011;4:211-22.

31. Nakanishi K, Homma S, Han J, et al. Usefulness of Tricuspid Annular Diameter to Predict Late Right Sided Heart Failure in Patients With Left Ventricular Assist Device. Am J Cardiol 2018;122:115-20. 